\title{
GMR
}

\section{Prospective uses of recombinant Lactococcus lactis expressing both listeriolysin 0 and mutated internalin A from Listeria monocytogenes as a tool for DNA vaccination}

\author{
M.S.P. De Azevedo', C. Santos Rocha', V.B. Pereira' ${ }^{1}$, A.F. De Oliveira Junior ${ }^{1}$, \\ C.S. De Sousa', V. Azevedo', J.G. LeBlanc ${ }^{2}$, J.M. Chatel ${ }^{3}$ and A. Miyoshi ${ }^{1}$ \\ 'Laboratório de Genética Celular e Molecular, Instituto de Ciências Biológicas, \\ Universidade Federal de Minas Gerais, Belo Horizonte, MG, Brasil \\ ${ }^{2}$ CERELA-CONICET, Chacabuco 145, Tucuman, Argentina \\ ${ }^{3}$ Institut National de la Recherche Agronomique, MICALIS (UMR 1319), \\ Domaine de Vilvert, Jouy-en-Josas, France \\ Corresponding author: M.S.P. De Azevedo \\ E-mail: marcela.spachecoazevedo@gmail.com
}

Genet. Mol. Res. 14 (4): 18485-18493 (2015)

Received September 25, 2015

Accepted October 16, 2015

Published December 23, 2015

DOI http://dx.doi.org/10.4238/2015.December.23.36

\begin{abstract}
In this study, Lactococcus lactis was engineered to express mutated internalin $A$ on its surface and to secrete large amounts of listeriolysin O (LLO) in order to improve its potential as a vehicle for DNA vaccination. Western blotting experiments demonstrated that the bacterium expressed LLO in both the cytoplasmic and extracellular compartments, with higher quantities found in the culture supernatants. A hemolytic assay showed that the recombinant strain secreted $250 \mathrm{ng}$ active LLO/mg total protein. This mInIA/LLO-producing strain of $L$. lactis may be used as an alternative tool in DNA vaccination against a number of infectious diseases or in cancer therapy.
\end{abstract}

Key words: Lactic acid bacteria; Lactococcus lactis; Listeria monocytogenes; Listeriolysin O; Mutated internalin A 


\section{INTRODUCTION}

The strategy of using generally recognized as safe bacteria, such as the very important lactic acid bacteria Lactococcus lactis, as a vector for delivering therapeutic plasmids has gained the attention of many scientists. Lactococcus lactis has been proposed as a being a safe and effective vaccine platform for the delivery of therapeutic molecules to the immune system (Nouaille et al., 2003; Pontes et al., 2011). It has been shown that wild-type (wt) L. lactis can deliver DNA vaccines both in vitro and in vivo after oral inoculation of mice (Guimarães et al., 2005; Chatel et al., 2008). However, the ratio of DNA transferred to mammalian cells was quite low. This low efficiency may be related to the transient nature of $L$. lactis, which may prevent an optimal interaction with intestinal epithelial cells in the gastrointestinal tract and therefore may prevent DNA delivery. Thus, strains of $L$. lactis expressing recombinant virulent genes were recently constructed in an attempt to improve their capacity to persist in the gastrointestinal tract and thus deliver larger amounts of DNA vaccines (Innocentin et al., 2009). Guimarães et al. (2005) explored the potential use of recombinant $L$. lactis strains engineered to express invasins naturally produced by enteropathogenic species, such as internalin A ( $\operatorname{ln|A}$ ) from the food-borne pathogen Listeria monocytogenes. InIA is a sortase anchored cell wall protein that is composed of 800 amino acids and mediates bacterial entry into mammalian epithelial cells (Vazquez-Boland et al., 2001). InlA from L. monocytogenes can invade the mucosal surfaces of the small intestine and interact with the Peyer's patch-based immune system, including intestinal dendritic cells, which are potent antigen-presenting cells (Lecuit et al., 1997). The InIA-expressing L. lactis ( $L L-I n I A+)$ strain was shown to invade Caco-2 cells in vitro and intestinal cells in vivo after oral immunization in guinea pigs. Furthermore, the LL-InIA+ strain could deliver a functional eukaryotic green fluorescent protein gene into epithelial Caco-2 cells more efficiently than the wt lactococci, demonstrating that this strain may be a good tool for gene delivery (Guimarães et al., 2005). However, use of the LL-InIA+ strain presented some bottlenecks, as InIA can only bind to its receptor (E-cadherin) in guinea pigs or transgenic mice, making in vivo studies laborious and/or expensive (Wollert et al., 2007). An L. lactis strain producing a mutated form of InIA (LL-mInIA+), which can bind to E-cadherin from conventional mice and humans, was recently reported by our research group. Mutated internalin A ( $\mathrm{mln} \mid \mathrm{A})$ gave $L$. lactis an invasive status, improving its interaction with intestinal epithelial cells and leading to a higher level of DNA transfer in vitro (de Azevedo et al., 2012). Since the use of LL-mInIA+ in vivo did not statistically increase DNA delivery, we constructed another strain that could express both $\mathrm{mInIA}$ and another virulence factor from L. monocytogenes, hemolysin listeriolysin O (LLO). In this pathogen, LLO expression was up-regulated at low $\mathrm{pH}$ (approximately $\mathrm{pH} 5.5$ ) inside the phagosome, which then oligomerized to form a pre-pore complex, rupturing the Listeria-containing phagosome (Cossart and Toledo-Arana, 2008). Therefore, the expression of LLO in L. lactis may facilitate escape of the plasmid DNA from the phagosome into the nucleus. Thus, cellular expression of the transfected gene may show increased efficiency as suggested by the fact that LLO incorporated in an anionic liposome effectively enhanced plasmid DNA delivery (Lorenzi and Lee, 2005). In addition to increasing plasmid transfer, it was demonstrated that LLO-containing $\mathrm{pH}$-sensitive liposomes encapsulated with immunostimulatory $\mathrm{CpG}$ oligonucleotides could efficiently deliver ovalbumin antigen to the cytosol of antigen-presenting cells and stimulate cytotoxic $T$ lymphocytes, driving Th1-type immune responses (Andrews et al., 2012). Another research group demonstrated that LLO-liposome-mediating cytosolic delivery of antigens in vivo enhanced antigen-specific cytotoxic 
T lymphocyte frequency, conferring tumor protection (Mandal and Lee, 2002). Thus, in the present study, a food-grade L. lactis NZ9000 strain was engineered to constitutively express both mInIA and LLO. This strain may be used as a vehicle for DNA vaccination in the future.

\section{MATERIAL AND METHODS}

\section{Bacterial strains, media, and growth conditions}

Bacterial strains and plasmids used in this study are listed in Table 1. Lactococcus lactis NZ9000 was used as the host strain for the pLL31 plasmid, which was provided by MORU VAZE Company, Paris, lle-de-France, France. Electrocompetent $L$. lactis strains were prepared and transformed with the corresponding pLL31 expression vector and plated onto M17 agar supplemented with $0.5 \%$ glucose and $10 \mathrm{mg} / \mathrm{mL}$ erythromycin. Colonies were screened by polymerase chain reaction analysis. Procedures for DNA manipulation have been described elsewhere (Sambrook et al., 1989).

\begin{tabular}{|c|c|c|}
\hline Strain/plasmid & Relevant characteristics & Source/reference \\
\hline \multicolumn{3}{|l|}{ Bacterial strains } \\
\hline Lactococcus lactis MG1363 (LL wt) & $\begin{array}{l}\text { A derivative of } L \text {. lactis MG1363 } \\
\text { wild type strain generated by the } \\
\text { integration of the NisRK genes }\end{array}$ & 26 \\
\hline $\begin{array}{l}\text { Lactococcus lactis expressing both } \\
\text { mutated internalin } A(\mathrm{mln} \mid \mathrm{A}) \text { and } \\
\text { Lysteriolisin } \mathrm{O}(\mathrm{LLO})(\mathrm{LL}-\mathrm{m}|\mathrm{n}| \mathrm{A}+\mathrm{LLO}+)\end{array}$ & L. lactis NZ9000 strain containing pLL31 plasmid & This work \\
\hline \multicolumn{3}{|l|}{ Plasmids } \\
\hline pLL31 & $\begin{array}{l}\text { L. lactis-Escherichia coli shuttle vector carrying } \\
\text { the mInIA gene under the control of the constitutive } \\
\text { PrfA promoter protein and harboring the native cell } \\
\text { wall anchoring signal and hlyA gene (encoding LLO), } \\
\text { containing its constitutive promoter and own signal peptide. }\end{array}$ & MORU VAZE Company \\
\hline
\end{tabular}

\section{Detection of recombinant mInIA by flow cytometry analysis}

Bacterial cells were collected and resuspended in phosphate-buffered saline containing $0.5 \%$ bovine serum albumin (BSA) (Sigma, St. Louis, MO, USA) and $10 \mathrm{mg} / \mathrm{mL}$ monoclonal antibody anti-mInIA. After incubation at $4^{\circ} \mathrm{C}$, the samples were centrifuged and the pellets were resuspended in phosphate-buffered saline containing $0.5 \% \mathrm{BSA}$ and fluorescein isothiocyanateconjugated AffiniPure Fab fragment goat anti-mouse IgG $(\mathrm{H}+\mathrm{L})$ (Jackson ImmunoResearch, West Grove, PA, USA). Bacteria were then fixed in $2 \%$ paraformaldehyde, and flow cytometry was performed using an Accuri C6 Flow Cytometer ${ }^{\circledR}$ System (BD Biosciences, Franklin Lakes, NJ, USA). Fluorescein isothiocyanate fluorescence was measured using two-color excitation. Data analysis was performed using Accuri CFlow Software.

\section{Investigation of LLO production by western blot analysis}

Protein sample preparation from $L$. lactis cultures was performed as previously described (Le Loir et al., 1998) and then used for immunodetection. The supernatant as well soluble and insoluble cytoplasmic protein extracts were collected, which were assayed for the presence of LLO. 
Sodium dodecyl sulfate-polyacrylamide gel electrophoresis was performed and the gels were blotted against a nitrocellulose membrane and then blocked overnight at $4^{\circ} \mathrm{C}$ in $5 \%$ BSA. Primary anti-LLO antibody (Abcam, Cambridge, UK) and secondary goat anti-rabbit IgG ( $\gamma$ heavy chain-specific) were used at 1:15,000 and 1:20,000 dilutions in 5\% BSA, respectively. Blots were then scanned and the recombinant LLO (rLLO) signals were analyzed using a commercial protein standard.

\section{Assessment of rLLO hemolytic activity}

To quantify LLO and verify whether LLO was being produced in an active form, the hemolytic activity of protein preparations was examined. First, total protein was quantified using a Bradford assay, and assay buffer $(A B)$ was prepared to give a $1 \%$ red blood cell solution diluted in low pH AB (LpHAB) (125 mM NaCl, $35 \mathrm{mM}$ dibasic sodium phosphate, $0.5 \mathrm{mg} / \mathrm{mL}$ BSA, pH 5.5). A sample of $1 \mathrm{mg}$ total protein derived from culture supernatants, $0.25 \mathrm{mg}$ total protein from soluble and insoluble cytoplasmic extracts, and $50 \mathrm{ng} / \mathrm{mL}$ rLLO diluted in LpHAB as well as a positive control $(40 \% \mu \mathrm{L} \mathrm{AB}$ solution, $50 \% \mathrm{LpHAB}, 10 \%$ Triton) were added to a 96 -well microtiter $\mathrm{V}$-bottom plate (Edge Bio). Negative control (LpHAB buffer) was also included. Samples were serially diluted two times and plate was incubated at $37^{\circ} \mathrm{C}$. After spin down, $100 \mu \mathrm{L}$ supernatant were transferred to a flat-bottomed microtiter plate (EdgeBio, Gaithersburg, MD, USA) and absorbance was measured at $540 \mathrm{~nm}$ in a spectrophotometer. Data were quantitatively analyzed using a standard curve, which was fitted based on rLLO.

\section{Statistical analyses}

Statistical significance between groups was calculated using one-way analysis of variance, followed by the Bonferroni post-test. Values of $\mathrm{P}<0.05$ were considered significant.

\section{RESULTS}

\section{Lactococcus lactis expressed mutated internalin A on its surface}

In this study, we proposed two approaches for enhancing plasmid transfer both in vitro and in vivo by expressing two major immunodominant antigens in L. lactis, LLO and mInIA, derived from L. monocytogenes. After transformation of the L. lactis NZ9000 strain with the pLL31 plasmid by electroporation, flow cytometry analyses were performed to detect recombinant mInIA. Plasmid pLL31 harbors native hlyA gene (encoding LLO) containing its constitutive promoter, a signal peptide, and a ribosomal binding site. It also contains the mutated internalin A open reading frame, the constitutive PrfA promoter protein, and the cell wall-anchoring signal. Lactococcus lactis expressing native InIA, wt L. lactis NZ9000, and the strain constructed in this study producing both LLO and $\mathrm{m} \ln \mid \mathrm{A}$ (LL-mInIA+LLO+) were incubated with monoclonal mouse anti-InIA antibody and then with fluorescein isothiocyanateconjugated AffiniPure Fab fragment goat anti-mouse $\lg G(\mathrm{H}+\mathrm{L})$. Data was analyzed by flow cytometry. As shown in Figure 1, strains producing mInIA (blue and yellow peaks) or native InIA (red peak) significantly shifted the distribution curve compared to the wt strain (black peak). Fluorescence intensity obtained for the mInIA-producing strain was 2-fold higher compared to the wt strain, while LL-InIA expression of recombinant protein was 4-fold higher. This assay confirmed the expression of mInIA on the surface of $L$. lactis. 


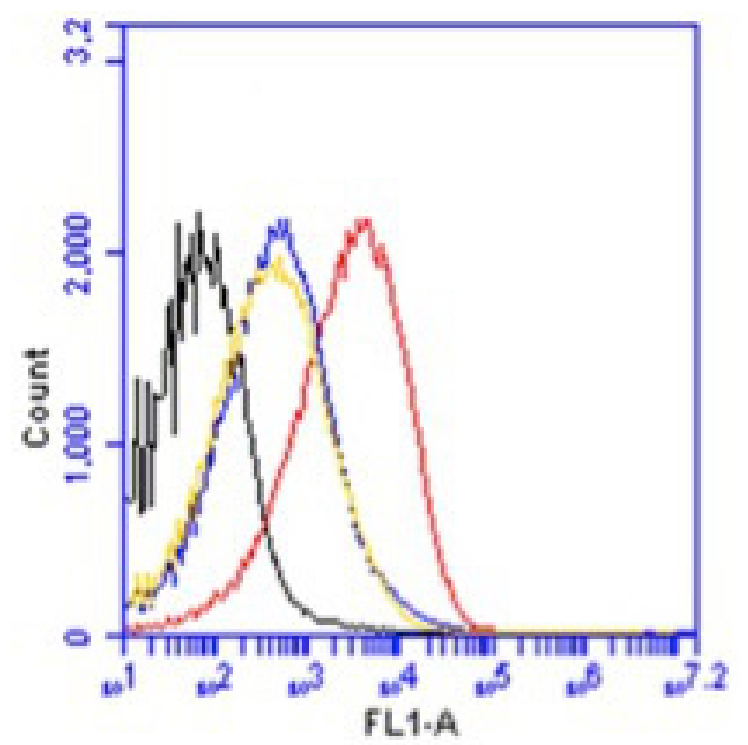

Figure 1. Detection of mutated InIA at L.lactis surface by fluorometry analysis. wt lactococci (black peak, negative control) or recombinant Lactococcus lactis expressing Listeria monocytogenes Internalin A $(\operatorname{In} \mid \mathrm{A})$ (red peak, positive control), mutated InIA (mInIA) (blue peak) or both Listeriolysin (LLO) and mInIA (yellow peak).

\section{Listeria monocytogenes LLO was constitutively produced and secreted by L. lactis}

After confirming mInIA expression on the L. lactis surface, western blotting experiments were conducted to detect rLLO in the cytoplasmic and extracellular cell compartments. Strains (wt and LL-mInIA+LLO+) were grown for protein extraction and both supernatant and cytoplasmic proteins were collected and subjected to sodium dodecyl sulfate-polyacrylamide gel electrophoresis. Gels were then blotted against a nitrocellulose membrane, which was incubated with primary antiLLO antibody and secondary goat anti-rabbit IgG ( $\gamma$ heavy chain-specific). LLO signals were then determined. Analysis of stationary-phase cell lysates revealed a band in accordance with the molecular weight of LLO (58 kDa), in both the soluble and insoluble cytoplasmic protein fractions as well as in the culture supernatants, with a large amount of recombinant protein found in this compartment (Figure 2). LLO was not detected in protein extracts from the supernatant and from the cytoplasmic compartments derived from the wt strain.

\section{Recombinant LLO was produced as an active pore-forming toxin in L. lactis}

Subsequently, a hemolytic assay was conducted to determine whether LLO was produced as an active protein. The culture supernatant and cytoplasmic proteins derived from both the wt and LL-mInIA+LLO+ strains were extracted and then used in the assay. Purified rLLO was used as a standard to measure protein activity and to quantify the amount of LLO produced by L. lactis. Either rLLO or protein preparations from wt or the L. lactis strain producing mInIA-LLO were incubated with red blood cells. Next, hemolytic activity was measured at $540 \mathrm{~nm}$ using a spectrophotometer. LLO production was quantified using the same approach. As shown in Figure 3 , protein extracts (both soluble and insoluble) as well as culture supernatants derived from 
recombinant $L$. lactis showed strong hemolytic activity compared to the negative control (LLwt). We also observed that LLO from the insoluble compartment has pore-forming activity similar to LLO derived from the soluble fraction. Furthermore, the highest hemolytic activity was observed in the culture supernatant. Moreover, nearly $250 \mathrm{ng} / \mathrm{mg}$ total protein was found to be constitutively secreted by $L$. lactis. This hemolytic assay clearly revealed that LLO is well-secreted in L. lactis as a highly active pore-forming toxin.

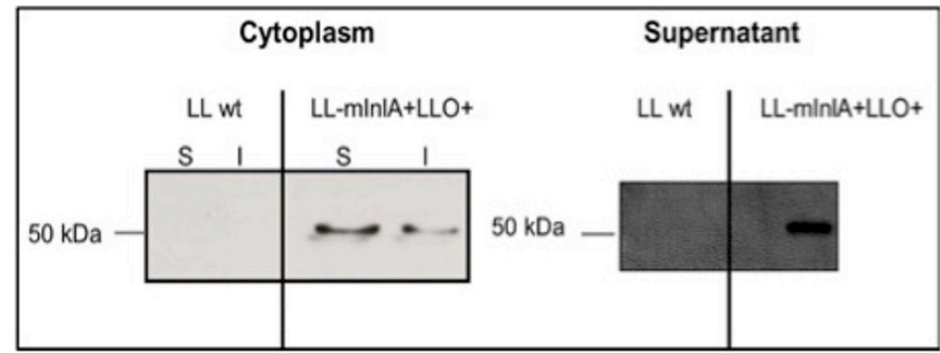

Figure 2. Western blot demonstrating the cytoplasmic and extracellular expression of Lysteriolisin $O$ (LLO) in Lactococcus lactis NZ9000. Protein extracts of recombinant or non-recombinant $L$. lactis strain were prepared from cell (left) and supernatant (right) fractions. LLwt: L. lactis NZ9000, LL-mInIA+LLO+: L. lactis expressing both Listeria monocytogenes mutated InIA and LLO, S: soluble cytoplasmic fraction, I: Insoluble cytoplasmic fraction.

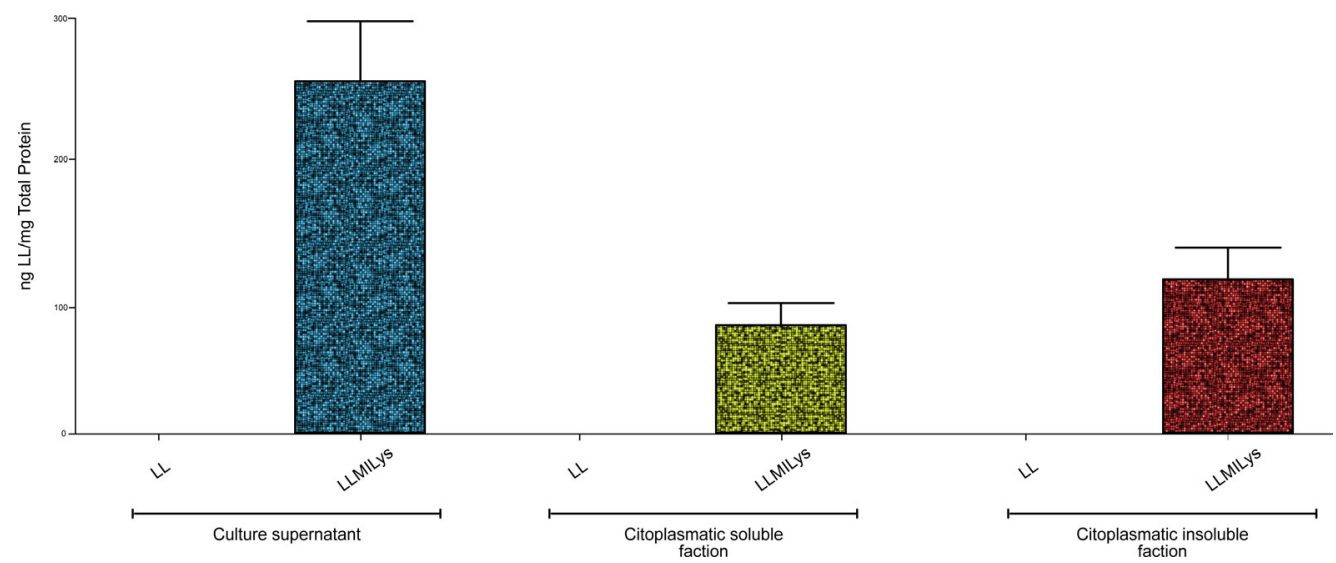

Figure 3. Haemolytic activity of Listeriolysin O (LLO) expressed in Lactococcus lactis. Sheep red blood cells were incubated with cytoplasmic extracts and culture supernatants to evaluate LLO pore forming capacity. LLwt: L. lactis NZ9000, LLMILys: L. lactis expressing both Listeria monocytogenes mutated InIA and LLO, S: soluble cytoplasmic fraction, I: Insoluble cytoplasmic fraction.

\section{DISCUSSION}

Attenuated strains of $L$. monocytogenes have been proposed for use as vehicles to deliver therapeutic plasmids to eukaryotic cells. This bacterium can induce its own uptake into nonphagocytic mammalian cells by expressing InIA and forming pores in phagolysosomal membranes through the production of LLO, facilitating DNA plasmid transfer to the mammalian cell nucleus (Lecuit et al., 1997; Tangney and Gahan, 2010). 
Several preclinical studies have demonstrated the usefulness of $L$. monocytogenes for intracellular gene delivery. Furthermore, this vector was shown to be relatively safe and efficacious in some clinical trials (Tangney and Gahan, 2010). However, concerns that attenuated vectors may restore their ability to replicate and cause disease in patients have been expressed. This concern should be considered before administration in infants and immunocompromised individuals. The reversion to virulence, preexisting immunity, and reactogenicity will always remain major concerns when pathogenic species are considered as vaccine platforms (Schoen et al., 2004).

Thus, the scientific community has recently explored the use of non-pathogenic bacteria, such as L. lactis, as prophylactic or therapeutic tools and, more recently, as DNA delivery vehicles for genetic immunization (Wells and Mercenier, 2008; Pontes et al., 2011). Chatel et al. (2008) showed that $L$. lactis could deliver a plasmid to epithelial cells of the intestinal membrane in conventional mice. Additionally, it was demonstrated that $L$. lactis can be used as a vector for genetic immunization; however, the plasmid transfer ratio was low. The transient physiology of $L$. lactis was considered to be the cause of this limitation. Thus, recent studies have focused on the construction of recombinant invasive lactococci strains to increase plasmid DNA delivery (Innocentin et al., 2009; Pontes et al., 2011; de Azevedo et al., 2012). Therefore, a mutated form of InIA that can bind to murine E-cadherin, $\mathrm{mInIA}$, was successfully expressed in $L$. lactis, facilitating in vivo studies. de Azevedo et al. (2012) demonstrated that the expression of mInlA on the L. lactis surface improved its invasive capacity in experiments performed using intestinal epithelial cells. In this study, we engineered $L$. lactis that expressed $\mathrm{mlnIA}$, as well as an additional virulent factor of $L$. monocytogenes, LLO, to facilitate plasmid escape to the nucleus, increasing production of the gene of interest. Flow cytometry analysis showed that mInIA was properly expressed on the $L$. lactis surface. The antibody used in the assay was specific for native InIA, which may explain the decreased detection of $\mathrm{mInIA}$. After confirming mInIA expression, evaluated the production of LLO by L. lactis. Western blot analysis revealed dual localization of LLO, which was found in both extracellular and intracellular compartments. Interestingly, half of the LLO was found in the soluble fraction, with a large amount secreted into the extracellular medium. The other half was detected in the insoluble fraction, demonstrating that LLO was attached to the cell envelope. This may have occurred because of the presence of inclusion bodies. It is known that recombinant proteins expressed in L. lactis may be inadequately stable or show low solubility, leading to improper protein folding, inclusion body formation, and protein degradation (Le Loir et al., 2005). Thus, it is possible that a post-translational process limits LLO secretion, such as insoluble aggregate formation.

The hemolytic assay demonstrated that the LLO protein was produced as an active poreforming toxin. This is a very important aspect when considering further study of this strain, as it ensures that phagosomal membranes will be disrupted, facilitating plasmid escape. Recently, Vadia et al. (2011) showed that LLO can serve as an invasion factor sufficient to induce the internalization of noninvasive Listeria innocua. This may be another advantageous feature of using the L. lactis mInIA-LLO-producing strain to establish more intimate contact with host cells from the gastrointestinal tract and then promote higher plasmid transfer after oral inoculation. Other research groups have reported the use of recombinant L. lactis expressing recombinant LLO for vaccination. Lactococcus lactis was engineered to produce LLO induced using an expensive peptide named nisin, or with the gene chromosomally integrated as a vaccine against listeriosis (Bahey-El-Din et al., 2008; Bahey-El-Din et al., 2010). LLO was engineered in this study to be constitutively expressed, which may facilitate its commercialization and lower purification costs compared with the other LLO-producing strain of $L$. lactis in which nisin is required to induce gene expression (Wollert et al., 2007). 
The use of $L$. lactis expressing both virulent factors from L. monocytogenes, including mInIA and LLO, as a tool for delivering DNA plasmids may represent an efficient strategy with significant applications in basic research, cancer therapy, or vaccinology. This strain may also be applied as a source of pure LLO because L. lactis produces neither inclusion bodies nor endotoxins, which differs from recombinant Escherichia coli expressing LLO containing lipopolysaccharide in its outer membrane (Giammarini et al., 2003). Finally, we are currently evaluating the DNA transfer capacity of this novel $L$. lactis strain in experiments performed with intestinal epithelial cells in vitro. We will then measure DNA transfer in vivo after oral inoculation of the strain into conventional BALB/c mice.

\section{Conflicts of interest}

The authors declare no conflict of interest.

\section{ACKNOWLEDGMENTS}

Research funded by the European Community's Seventh Framework Programme (\#FP7/2007-2013), under grant agreement \#215553-2, and the French-Brazilian CAPES COFECUB project \#720/11. We thank Dr. Catherine Grillot Courvalin (Associate Professor at the Pasteur Institute, Unit of Antibacterial Agents, Paris, France) for providing the rLLO and valuable scientific input. We also thank the MORU VAZE Company for providing the pLL31 plasmid.

\section{REFERENCES}

Andrews CD, Huh MS, Patton K, Higgins D, et al. (2012). Encapsulating immunostimulatory CpG oligonucleotides in listeriolysin O-liposomes promotes a Th1-type response and CTL activity. Mol Pharm. 9: 1118-1125.

Bahey-El-Din M, Casey PG, Griffin BT and Gahan CG (2010). Efficacy of a Lactococcus lactis $\Delta$ pyrG vaccine delivery platform expressing chromosomally integrated hly from Listeria monocytogenes. Bioeng. Bugs. 1: 66-74.

Bahey-El-Din M, Griffin BT and Gahan CGM (2008). Nisin inducible production of listeriolysin O in Lactococcus lactis NZ9000. Microb. Cell. Fact. 29: 7-24.

Chatel JM, Pothelune L, Ah-Leung S, Corthier G, et al. (2008). In vivo transfer of plasmid from food-grade transiting lactococci to murine epithelial cells. Gene Ther. 15: 1184-1190.

Cossart P and Toledo-Arana A (2008). Listeria monocytogenes, a unique model in infection biology: an overview. Microbes Infect. 10: 1041-1050.

de Azevedo M, Karczewski J, Lefévre F, Azevedo V, et al. (2012). In vitro and in vivo characterization of DNA delivery using recombinant Lactococcus lactis expressing a mutated form of L. monocytogenes internalin A. BMC Microbiol. 12 : 299.

Giammarini C, Andreoni F, Amagliani G, Casiere A, et al. (2003). High-level expression of the Listeria monocytogenes listeriolysin $\mathrm{O}$ in Escherichia coli and preliminary characterization of the purified protein. Protein Expr. Purif. 28: 78-85.

Guimarães VD, Gabriel JE, Lefèvre F, Cabanes D, et al. (2005). Internalin-expressing Lactococcus lactis is able to invade small intestine of guinea pigs and deliver DNA into mammalian epithelial cells. Microbes Infect. 7: 836-844.

Innocentin S, Guimarães V, Miyoshi A, Azevedo V, et al. (2009). Lactococcus lactis expressing either Staphylococcus aureus fibronectin-binding protein A or Listeria monocytogenes internalin A can efficiently internalize and deliver DNA in human epithelial cells. Appl. Environ. Microbiol. 75: 4870-4878.

Le Loir Y, Gruss A, Ehrlich SD and Langella P (1998). A nine-residue synthetic propeptide enhances secretion efficiency of heterologous proteins in Lactococcus lactis. J. Bacteriol. 180: 1895-1903.

Le Loir Y, Azevedo V, Oliveira SC, Freitas DA, et al. (2005). Protein secretion in Lactococcus lactis: an efficient way to increase the overall heterologous protein production. Microb. Cell. Fact. 4: 4-2.

Lecuit M, Ohayon H, Braun L, Mengaud J, et al. (1997). Internalin of Listeria monocytogenes with an intact leucine-rich repeat region is sufficient to promote internalization. Infect. Immun. 65: 5309-5319.

Lorenzi GL and Lee KD (2005). Enhanced plasmid DNA delivery using anionic LPDII by listeriolysin O incorporation. J. Gene Med. 7: 1077-1085. 
Mandal M and Lee KD (2002). Listeriolysin O-liposome-mediated cytosolic delivery of macromolecule antigen in vivo: enhancement of antigen-specific cytotoxic T lymphocyte frequency, activity, and tumor protection. Biochim. Biophys. Acta 1563: 7-17.

Nouaille S, Ribeiro LA, Miyoshi A, Pontes D, et al. (2003). Heterologous protein production and delivery systems for Lactococcus lactis. Genet. Mol. 2: 102-111.

Pontes DS, de Azevedo MS, Chatel JM, Langella P, et al. (2011). Lactococcus lactis as a live vector: heterologous protein production and DNA delivery systems. Protein Expr. Purif. 79: 165-175.

Sambrook J, Fritsch EF and Maniatis T (1989). Molecular cloning: A laboratory manual: 2nd ed. Cold Spring Harbor Laboratory Press, Cold Spring Harbor.

Schoen C, Stritzker J, Goebel W and Pilgrim S (2004). Bacteria as DNA vaccine carriers for genetic immunization. Int. J. Med. Microbiol. 294: 319-335.

Tangney M and Gahan CG (2010). Listeria monocytogenes as a vector for anti-cancer therapies. Curr. Gene Ther. 10: 46-55.

Vadia S, Arnett E, Haghighat AC, Wilson-Kubalek EM, et al. (2011). The pore-forming toxin listeriolysin O mediates a novel entry pathway of L. monocytogenes into human hepatocytes. PLoS Pathog. 7: e1002356.

Vazquez-Boland JA, Kuhn M, Berche P, Chakraborty T, et al. (2001). Listeria pathogenesis and molecular virulence determinants. Clin. Microbiol. Rev. 14: 584-640.

Wells JM and Mercenier A (2008). Mucosal delivery of therapeutic and prophylactic molecules using lactic acid bacteria. Nat. Rev. Microbiol. 6: 349-362.

Wollert T, Pasche B, Rochon M, Deppenmeier S, et al. (2007). Extending the host range of Listeria monocytogenes by rational protein design. Cell 129: 891-902. 